\title{
Fatores associados à escolha da segunda especialidade entre concluintes da residência em clínica médica
}

\section{Factors associated with the choice of a second specialty among internal medicine residency graduates}

\author{
Marco Felipe Macêdo Alves ${ }^{1}$ (D) $\mid$ marcofma7@gmail.com \\ Jardelina Brena Rocha Leite ${ }^{1}$ (D) brenaarochaa17@gmail.com \\ Norma Arteiro Filgueira' (D) norma.arteiro@hotmail.com
}

\begin{abstract}
RESUMO
Introdução: Após a residência em clínica médica, a maioria dos concluintes opta por se submeter a um novo processo seletivo para obter uma segunda especialidade. O fenômeno da especialização precoce é incentivado já na graduação. Apesar disso, a demanda por médicos generalistas está em crescimento.
\end{abstract}

Objetivo: Este estudo teve como objetivo investigar os fatores que levam o clínico recém-formado a realizar uma nova residência.

Método: Trata-se de um estudo transversal que analisou as respostas dos concluintes do Programa de Residência Médica em Clínica Médica (PRM-CM) realizado em 2020 no estado de Pernambuco. Os concluintes do PRM-CM, por meio de um questionário disponibilizado de forma on-line pelo Google Forms, responderam a perguntas sobre aspectos sociais, a graduação, a residência médica e intenções para a carreira futura.

Resultado: Dos 104 participantes possíveis, houve 81 respostas (77,88\%). Desse total final, 66,67\% eram do sexo feminino, 69,14\% tinham se graduado em universidades públicas, e 50,62\% já haviam iniciado o PRM-CM logo após a graduação. Quanto à escolha de especialidade, 51,85\% responderam ter decidido no segundo ano de residência, e 80,25\% afirmaram ter se submetido ao processo seletivo para a segunda especialidade logo após o PRM-CM. A carreira mais escolhida foi cardiologia (20\%). Os fatores mais associados à escolha de especialidade foram, de acordo com as médias na escala de Likert, "trabalho em ambiente ambulatorial", "acompanhamento de pacientes por longo período" e "mais contato com pacientes".

Conclusão: Até onde se pôde investigar na literatura, este é o primeiro estudo brasileiro a abordar as escolhas de especialidade após o PRM-CM. Foi possível identificar os motivos mais importantes para escolher uma segunda especialidade entre os concluintes desse PRM em Pernambuco, em 2020. Mais estudos são necessários para tecer correlações entre os fatores de escolha com a especialidade escolhida.

Palavras-chave: Residência Médica; Medicina Interna; Especialidades Médicas; Educação Médica; Escolha da Profissão.

\section{ABSTRACT}

Introduction: After the residency in Internal Medicine, most graduates choose to undergo a new selection process to obtain a second specialty. The phenomenon of early specialization is encouraged as early as in the undergraduate course. Despite this, the demand for general practitioners is growing.

Objective: To investigate the factors that lead the newly graduated clinician to undertake a new residency.

Method: This is a cross-sectional study that analyzes the responses of Internal Medicine residency graduates from the state of Pernambuco in 2020, through a questionnaire available online by Google Forms, containing questions about social aspects, undergraduate medical course, Medical Residency and intentions for the future career.

Results: There were 81 responses of the 104 possible participants (77.88\%). Most of these were female (66.67\%), graduated from public universities (69.14\%) and had already started the Internal Medicine residency shortly after graduation (50.62\%). Regarding the specialty choice, $51.85 \%$ answered they had decided in the second year of residency, and $80.25 \%$ stated that they had undergone the selection process for the second specialty shortly after completing the Internal Medicine residency. The most often chosen career was Cardiology (20\%). The factors most often associated with the choice of specialty were, according to the means on the Likert scale, "work in an outpatient setting", "long-term patient followup", and "more contact with patients".

Conclusion: As far as it could be investigated in the literature, this was the first Brazilian study on specialty choices after the Internal Medicine residency. It was possible to identify the most important reasons for choosing a second specialty among the graduates of this Medical Residency program in Pernambuco in 2020. More studies are needed to establish correlations between the factors of choice with the chosen specialty.

Keywords: Medical Residency; Internal Medicine; Medical Specialties; Medical Education; Career Choice.

${ }^{1}$ Universidade Federal de Pernambuco, Recife, Pernambuco, Brasil.

Editora-chefe: Rosiane Viana Zuza Diniz

Editor associado: Jorge Carvalho Guedes

Recebido em 26/07/21; Aceito em 08/09/21.

Avaliado pelo processo de double blind review. 


\section{INTRODUÇÃO}

Clínica médica é a especialidade da medicina que trata do processo saúde-doença do adulto, atuando ainda na promoção da saúde e na prevenção de doenças, em âmbito ambulatorial, domiciliar ou hospitalar. A partir dela, deriva-se a maioria das especialidades clínicas'. Desse modo, a formação em clínica médica é pré-requisito para o ingresso nas residências em alergia e imunologia, angiologia, cancerologia clínica, cardiologia, endocrinologia, gastroenterologia, geriatria, hepatologia, hematologia e hemoterapia, nefrologia, pneumologia e reumatologia, podendo também ser requisito prévio obrigatório para endoscopia, nutrologia e medicina intensiva².

A escolha da especialidade médica começa, geralmente, nos primeiros semestres da graduação e pode sofrer mudanças ao longo do curso e da carreira profissional, tendo sido verificado, em estudos anteriores, que depende de múltiplas variáveis, como retorno financeiro, possibilidade de divisão entre trabalho e tempo livre, conhecimento mais amplo ou mais específico, influência de parentes médicos e/ou professores, e afinidade com a especialidade ${ }^{3}$.

$\mathrm{Na}$ literatura internacional, encontram-se alguns trabalhos que discutem os motivos para o clínico optar por se subespecializar. Com base nesses motivos, é possível afirmar que existe cada vez mais tendência desses profissionais à segunda especialização ${ }^{4,5}$, destacando-se como fatores importantes estilo de vida mais controlável e experiências na residência ${ }^{6}$, e a intenção de acompanhamento dos pacientes em longo prazo e o maior retorno financeiro ${ }^{4,7}$.

Já na literatura brasileira, não há estudos diretamente relacionados às decisões após a residência em clínica médica. Apesar disso, existem aqueles que abordam a graduação médica, nos quais se nota que o modelo de ensino tradicional nas faculdades de Medicina parece incentivar a especialização precoce, seja pelo conhecimento fragmentado por especialidades, seja pela atenção à saúde individual maior que à saúde coletiva, seja pela criação do imaginário de que as especialidades mais complexas têm maiores prestígio social e retorno financeiro ${ }^{8}$.

Tendo em vista os impasses apontados, investigaremos, neste estudo, os fatores que levam à escolha de uma segunda especialidade pelos concluintes da residência médica em clínica médica do estado de Pernambuco. Além disso, traçaremos o perfil do concluinte da residência em clínica médica do estado de Pernambuco em 2020, determinaremos a frequência de opção por um segundo Programa de Residência Médica (PRM) após a conclusão da residência em clínica médica e analisaremos as razões para a não realização de um segundo processo seletivo no ano vigente.

\section{MÉTODO}

Trata-se de um estudo analítico, observacional, quantitativo, do tipo transversal, construído a partir de um questionário aplicado em meio eletrônico, na internet, na plataforma Google Forms, aos concluintes do Programa de Residência Médica em Clínica Médica (PRM-CM) de Pernambuco, em fevereiro de 2020.

O questionário contém os seguintes blocos de perguntas:

- Informações gerais (gênero, local e ano de conclusão do curso de Medicina, universidade pública ou privada, com quem residiu durante a residência médica, trabalhos anteriores e filhos).

- Informações sobre o PRM-CM que concluiu em fevereiro de 2020 (local, ser ou não em sua cidade de procedência, se o serviço tem ou não setor de emergência).

- Informações sobre próxima a residência médica (se realizou processo seletivo para residência médica para o ano de 2020, para qual(is) programa(s) se inscreveu, motivações para escolha da segunda especialidade e momento de escolha). Para os fatores que motivaram a escolha da residência médica, foi utilizado o modelo de escala Likert, havendo, em cada item, cinco opções de resposta - discordo totalmente (1), discordo parcialmente (2), indiferente (3), concordo parcialmente (4) e concordo totalmente (5) - para que o voluntário assinalasse aquela mais adequada ao seu perfil.

- Motivos para não realizar um segundo processo seletivo ao término do PRM-CM.

A relação nominal dos concluintes foi enviada pela Comissão Estadual de Residência Médica de Pernambuco (Cerem-PE), contendo 105 concluintes do PRM-CM de 2020. Desse total, excluiu-se uma participante porque o término da residência dela foi postergado para agosto por conta da licença-maternidade. Restaram, então, 104 concluintes, aos quais foi enviado o link para a pesquisa por meio do WhatsApp Messenger.

Foram permitidas respostas até o dia 5 de janeiro de 2021. A assinatura do Termo de Consentimento Livre e Esclarecido (TCLE) ocorreu também de forma on-line, antes de os participantes terem acesso ao formulário da pesquisa.

\section{Critérios de inclusão e exclusão}

- Critério de inclusão: ter concluído o PRM-CM em Pernambuco, em fevereiro de 2020. 
- Critério de exclusão: atraso no término do PRM-CM causado por trancamento, licença-maternidade ou afastamento por doença, ou quaisquer outros motivos não listados.

\section{Análise estatística}

Os dados foram agrupados sob a forma de medidas de tendência central e proporções. Para a análise dos fatores associados à escolha da especialidade, agrupamos as respostas da escala Likert da seguinte forma:

- "Indiferente", "discordo parcialmente" e "discordo totalmente": sem influência na escolha.

- "Concordo totalmente" e "concordo parcialmente": com influência na escolha.

As respostas foram transformadas em planilhas do Microsoft Excel e analisadas por meio do programa $R$ para Windows, versão 3.2.2 - 2015 - R Foundation for Statistical Computing.

\section{Aspectos éticos}

A pesquisa obedece aos preceitos éticos da Resolução n ${ }^{\circ} 466 / 2012$ do Conselho Nacional de Saúde, envolvendo aprovação eletrônica do TCLE para a sua realização. $O$ anonimato dos voluntários foi garantido com o sigilo sobre os dados da pesquisa, com o acesso aos dados somente pelos pesquisadores participantes por meio de senha virtual, e pelo fato de os participantes não precisarem informar seus nomes no formulário de pesquisa. Não houve benefício direto aos voluntários, porém os dados coletados poderão contribuir para ações de planejamento estratégico dos PRM-CM.

$O$ projeto de pesquisa teve anuência da Cerem-PE e foi aprovado pelo Comitê de Ética em Pesquisa do Hospital das Clínicas da Universidade Federal de Pernambuco (HC-Ufpe): Certificado de Apresentação de Apreciação Ética (CAAE) no 39795920.0.0000.8807.

\section{RESULTADOS}

Dos 104 participantes possíveis, houve 88 respostas, das quais sete foram excluídas por serem duplicadas, restando, então, 81 respostas válidas, o que corresponde a 77,88\% de respondedores. A Tabela 1 discrimina as respostas válidas por serviço em que se realizou o PRM-CM. Houve participação de residentes oriundos de todos os serviços em que houve conclusão do PRM-CM do estado de Pernambuco em 2020.

A Tabela 1 contém as respostas relacionadas às características sociais e às variáveis acerca de graduação e residência. A maioria dos participantes foi do sexo feminino $(66,67 \%)$, não possuíam filhos $(91,36 \%)$ e realizaram graduação em universidades públicas $(69,14 \%)$.
A maior parte dos voluntários (74,07\%) finalizou a graduação em universidades do estado de Pernambuco, a maioria na Universidade de Pernambuco (UPE) - 28,04\%. Ademais, 50,62\% seguiram o caminho da especialização precoce - realizaram o PRM-CM logo após a graduação.

Por fim, predominaram os entrevistados que declararam ter trabalhado em unidades de saúde da família e/ou em emergências básicas antes do PRM-CM. Além disso, verificase que, durante a residência, houve número expressivo de voluntários que moravam com cônjuge, pais ou amigos.

A Tabela 2 mostra os fatores relacionados à escolha da segunda residência pelos concluintes do PRM-CM em Pernambuco. Observou-se que a maioria dos concluintes escolheu a especialidade durante o segundo ano da residência e que $80,25 \%$ optaram por prestar prova para uma nova especialidade, e 7,41\% fizeram prova para mais de uma especialidade. Além disso, dentre as opções de especialidade, as cinco mais escolhidas foram, em ordem decrescente: cardiologia (20,0\%), endocrinologia (15,56\%), nefrologia $(11,11 \%)$, geriatria $(11,11 \%)$ e pneumologia $(7,78 \%)$.

Tabela 1. Características dos 81 concluintes do Programa de Residência Médica em Clínica Médica de Pernambuco em 2020.

\begin{tabular}{lcc}
\hline \multicolumn{1}{c}{ Característica } & N & Frequência \\
\hline Gênero feminino & 54 & $66,67 \%$ \\
Possui filhos & 7 & $8,64 \%$ \\
$\begin{array}{l}\text { Graduação em universidade pública } \\
\begin{array}{l}\text { Graduação em universidades de } \\
\text { Pernambuco }\end{array}\end{array}$ & 56 & $69,14 \%$ \\
$\begin{array}{l}\text { Início da residência no primeiro ano } \\
\text { após a graduação }\end{array}$ & 60 & $74,07 \%$ \\
\hline
\end{tabular}

Trabalhos anteriores à residência

$\begin{array}{lcc}\text { Emergência básica } & 56 & 69,14 \% \\ \begin{array}{l}\text { Unidade de saúde da família (atenção } \\ \text { básica) }\end{array} & 42 & 51,85 \% \\ \text { Emergência referência } & 32 & 39,51 \% \\ \text { Unidade de terapia intensiva } & 19 & 23,46 \% \\ \text { Evolucionista } & 7 & 8,64 \% \\ \text { Outros }^{\text {a }} & 11 & 13,57 \%\end{array}$

Com quem residiu durante a residência?

\begin{tabular}{lcc} 
Cônjuge & 25 & $30,87 \%$ \\
Pais & 23 & $28,40 \%$ \\
Sozinho & 20 & $24,69 \%$ \\
Outro(s) parente(s) & 12 & $14,81 \%$ \\
Amigos & 1 & $1,23 \%$ \\
\hline
\end{tabular}

a Outros: domicílio (6,17\%), Forças Armadas (3,7\%), consultório privado $(2,47 \%)$, clínica de Diálise $(1,23 \%)$. 
Tabela 2. Fatores relacionados à escolha da segunda especialidade entre os concluintes do Programa de Residência Médica em Clínica Médica de Pernambuco.

\begin{tabular}{lcc}
\hline \multicolumn{1}{c}{ Momento em que escolheu a especialidade } & N & Frequência \\
\hline Graduação & 31 & $38,27 \%$ \\
Entre graduação e residência & 4 & $4,94 \%$ \\
Primeiro ano de residência & 4 & $4,94 \%$ \\
Segundo ano de residência & 42 & $51,85 \%$ \\
Fez prova para segunda residência & 65 & $80,25 \%$ \\
Fez prova para mais de uma especialidade & 6 & $7,41 \%$ \\
\hline Para qual especialidade fez prova? ${ }^{a}$ & & $20,0 \%$ \\
Cardiologia & 18 & $15,56 \%$ \\
Endocrinologia & 14 & $11,11 \%$ \\
Geriatria & 10 & $11,11 \%$ \\
Nefrologia & 10 & $7,78 \%$ \\
Pneumologia & 7 & $5,56 \%$ \\
Gastroenterologia & 5 & $4,44 \%$ \\
Oncologia & $4,44 \%$ \\
Cuidados paliativos & 4 & $16,67 \%$ \\
Outros & & $60,49 \%$ \\
Faria residência em clínica médica ainda que não fosse pré-requisito obrigatório & 4 & $100 \%$ \\
\hline Total $^{\text {Par }}$ & 15 & 49 \\
\hline
\end{tabular}

a. Para esse item, considerar o total de 90 , já que alguns participantes indicaram duas ou três respostas.

b. Outros: especialidades que atingiram entre um e três votos, em ordem decrescente: reumatologia, ano adicional em clínica médica, psiquiatria, medicina intensiva, infectologia, endoscopia, nutrologia, hepatologia e hematologia/hemoterapia.

Tabela 3. Fatores associados à escolha da especialidade após a residência em clínica médica.

\begin{tabular}{lccc}
\hline \multicolumn{1}{c}{ Fator } & Média & IC (95\%) & Influência \\
\hline Trabalho em ambiente ambulatorial & 4,16 & $3,91-4,41$ & $79,75 \%$ \\
Acompanhamento de pacientes por longo período & 4,08 & $3,83-4,33$ & $72,15 \%$ \\
Mais contato com pacientes & 3,97 & $3,75-4,19$ & $77,22 \%$ \\
Experiências positivas na residência & 3,82 & $3,56-4,08$ & $68,35 \%$ \\
Amplo campo de trabalho & 3,75 & $3,46-4,04$ & $58,23 \%$ \\
Maior parte do tempo trabalhando em equipe & 3,67 & $3,42-3,92$ & $55,13 \%$ \\
Melhor planejamento da rotina de trabalho & 3,67 & $3,43-3,91$ & $58,97 \%$ \\
Contato com ambiente acadêmico/universitário & 3,59 & $3,32-3,86$ & $56,96 \%$ \\
Experiências positivas na graduação & 3,57 & $3,25-3,89$ & $60,76 \%$ \\
Trabalho em ambiente hospitalar & 3,56 & $3,29-3,83$ & $56,96 \%$ \\
Mercado de trabalho & 3,54 & $3,29-3,79$ & $55,70 \%$ \\
Retorno financeiro & 3,20 & $2,96-3,44$ & $43,04 \%$ \\
Tempo Livre & 3,02 & $2,74-3,30$ & $37,97 \%$ \\
Influência de professores na graduação & 2,99 & $2,69-3,29$ & $34,18 \%$ \\
Possibilidade de realizar procedimentos & 2,76 & $2,42-3,10$ & $37,97 \%$ \\
Influência de preceptores na residência & 2,70 & $2,39-3,01$ & $30,38 \%$ \\
Maior parte do tempo trabalhando sozinho & 2,22 & $1,96-2,48$ & $11,39 \%$ \\
Menos contato com o fim de vida de pacientes & 1,89 & $1,63-2,15$ & $10,13 \%$ \\
Menor concorrência na prova & 1,58 & $1,37-1,80$ & $5,06 \%$ \\
\hline
\end{tabular}


A Tabela 3 mostra, em ordem decrescente das médias na escala de Likert, os fatores mais importantes para a escolha da segunda especialidade após a conclusão do PRM-CM e seus respectivos intervalos de confiança, além do percentual de respostas transformadas em "influência positiva", ou seja, às quais foram atribuídas as pontuações de 4 ou 5 na escala de Likert, representando, respectivamente, "concordo parcialmente" ou "concordo totalmente".

Outrossim, 16 voluntários (19,75\%) não realizaram a prova para um segundo processo seletivo em 2020. Desses, dois relataram não ter intenção de prestar prova no futuro: um afirmou que a vocação dele era a medicina interna, e o outro não apresentou justificativa. A Tabela 4 apresenta os motivos que levaram os 14 participantes restantes a adiar a realização de um novo processo seletivo.

\section{DISCUSSÃO}

Até onde foi possível investigar na literatura, este é o primeiro estudo brasileiro a analisar os motivos para decisão sobre a segunda especialidade após a conclusão da residência em clínica médica, além de investigar o perfil epidemiológico dos residentes e as razões para não realizar um novo processo seletivo no momento.

Assim, observamos que os principais fatores determinantes para escolha da segunda especialidade foram: 1. trabalho em ambiente ambulatorial; 2 . acompanhamento de pacientes por longo período; 3. mais contato com pacientes.

O clínico deve ter, em suas competências, capacidade de trabalhar em dois ambientes: hospitalar e ambulatorial ${ }^{9}$. O atual modelo hospitalocêntrico de residência médica, apesar de suprir a educação no modelo de atenção hospitalar em plantões e evoluções de pacientes internados, pode fragilizar o treinamento em ambiente ambulatorial. Mais recentemente, tem crescido uma tendência em aumentar a carga horária em ambulatório e valorizar a relação hospital-ambulatório para aprimorar a educação do clínico ${ }^{10}$. Essa necessidade pode ser corroborada pelo achado em nosso estudo de que o principal fator determinante para a escolha da segunda especialidade foi justamente o trabalho em ambiente ambulatorial, que é um dos principais campos de trabalho da maioria das especialidades clínicas. Dessa forma, um melhor treinamento em consultórios pode ofertar melhores clínicos e melhores especialistas para nossa sociedade.

Além disso, nota-se que os outros dois fatores tidos como mais importantes para a decisão sobre a segunda especialidade - acompanhamento de longo prazo e contato com pacientes - são provavelmente comuns à clínica médica e às especialidades clínicas. No estudo Demografia médica no Brasil 202011, é evidenciado que 90,9\% dos médicos
Tabela 4. Motivos para não realizar um novo processo seletivo no momento

\begin{tabular}{lcc}
\hline \multicolumn{1}{c}{ Motivo } & Respostas & Frequência \\
\hline Cansaço do período da residência & 11 & $78,57 \%$ \\
$\begin{array}{l}\text { Indefinição sobre qual especialidade } \\
\text { seguir }\end{array}$ & 7 & $50 \%$ \\
Condições financeiras & 5 & $35,71 \%$ \\
Permanecer perto de familiares & 4 & $28,57 \%$ \\
Cuidar de filhos & 2 & $14,29 \%$ \\
\hline Total & 14 & $100 \%$ \\
\hline
\end{tabular}

participantes da pesquisa trabalham em contato direto com pacientes. Isso pode evidenciar a necessidade de, na residência em clínica médica, treinamento referente à relação médico-paciente, à empatia e à comunicação, que são, assim como o atendimento ambulatorial, competências explicitadas nas últimas Matrizes de Competência em Clínica Médica9.

Para efeito de comparação, um estudo realizado em 2005 nos Estados Unidos revelou que os principais fatores que levavam os residentes de clínica médica a fazer uma subespecialidade foram relação de longo prazo com pacientes, campo de prática mais específico e cuidar de pacientes criticamente enfermos ${ }^{12}$.

Em nosso estudo, dos 81 participantes, 65 relataram ter feito prova para especialidade clínica $(80,25 \%)$ e outros 14 mencionaram ter a intenção de realizá-la no futuro $(17,28 \%)$, havendo, portanto, intenção de trabalhar em subespecialidades em $97,53 \%$ da população pesquisada. Esse número é superior ao evidenciado em estudo feito nos Estados Unidos, que revelou que $64,2 \%$ dos residentes do terceiro ano de clínica médica têm intenção de trabalhar em subespecialidades ${ }^{4}$. Igualmente, outro estudo estadunidense revelou que 59\% dos concluintes têm planos de realizar uma segunda especialidade ${ }^{5}$. Dessa forma, o resultado encontrado em nosso estudo está de acordo com outros trabalhos que evidenciam que a maioria dos concluintes da residência em clínica médica tende a realizar uma segunda especialidade. Apesar disso, nosso estudo encontrou que a maioria dos participantes teria cursado essa residência ainda que não fosse pré-requisito obrigatório para realizar a residência para a segunda especialidade, podendo evidenciar o reconhecimento da importância da clínica médica como base para a atuação nas especialidades.

Com relação ao perfil epidemiológico dos concluintes do PRM-CM do estado de Pernambuco em 2020, nota-se a predominância de mulheres (66,67\%), corroborando a participação feminina cada vez maior nos últimos anos na medicina brasileira ${ }^{11}$.

Além disso, a maioria dos concluintes de clínica médica declarou ter realizado graduação em universidades públicas, o 
que contrasta com dados do estudo Demografia médica no Brasil 2020, em que a maioria dos médicos participantes declarou ter realizado graduação em universidades privadas ${ }^{11}$. Existem dois fatores que podem explicar essa disparidade:

- A literatura mostra melhores resultados no Exame Nacional de Desempenho dos Estudantes (Enade) entre os estudantes de Medicina das universidades públicas $^{13}$, o que pode justificar os melhores desempenhos nos processos seletivos de residência médica.

- Na última década, houve um aumento de ofertas de fundos de financiamento estudantil nas escolas médicas privadas brasileiras ${ }^{14}$, e evidências apontam maior tendência a seguir carreira de médico generalista entre aqueles com maiores dívidas relacionadas à educação ${ }^{7}$.

Outrossim, a maioria dos participantes relatou ter realizado o processo seletivo para ingresso na residência em clínica médica logo após a graduação, o que mostra a tendência de especialização precoce do médico brasileiro ${ }^{15}$, aspecto decorrente, por vezes, de pressão própria ou social ${ }^{3}$. Outro dado encontrado em nosso estudo refere-se ao fato de a minoria ter filhos, o que pode corroborar a premissa de que uma população mais jovem opta pela especialização médica. A escolha decisiva da especialidade em nosso estudo ocorreu, na maioria das vezes, no segundo ano de residência, estando de acordo com outros estudos $^{5}$, porém um número significativo de participantes relatou ter escolhido a especialidade já na graduação.

Outro aspecto que merece destaque é que $80,25 \%$ dos participantes já se submeteram a um novo processo seletivo para uma segunda especialidade logo após o fim da residência em clínica médica, mostrando também a subespecialização precoce. Isso pode ocorrer por diversos fatores, como: 1. a não valorização do clínico pela sociedade e pelo mercado de trabalho, muitas vezes não se fazendo distinções entre o clínico e o médico generalista ${ }^{16} ; 2$. a crescente intenção médica em ter uma área de conhecimento mais restrita ${ }^{11}$, seja pelo receio de não conseguir se manter atualizado sobre uma ciência cada vez mais célere, seja por não desejar realizar um atendimento holístico (o que demanda tempo e dedicação em cada consulta, reduzindo a quantidade de pacientes atendidos por turno). Em um mundo em que a medicina se torna cada vez mais fragmentada, há uma demanda reprimida crescente pelo clínico, já que é o profissional que consegue resolver a maioria dos problemas - antes de ser necessária a consulta a um subespecialista - e que consegue conciliar as diversas prescrições e orientações dadas aos pacientes multimórbidos, em diversos ambientes de trabalho ${ }^{17}$.
Quanto às razões para não se submeter a um processo seletivo para uma segunda especialidade no momento, a principal resposta foi "cansaço do período da residência", o que está de acordo com outros estudos em que se revela a quantidade de residentes que adquirem a síndrome de burnout durante a pós-graduação ${ }^{18-20}$, o que pode vir a influenciar negativamente tanto a escolha quanto o momento de realizar uma nova residência médica.

Como limitações do nosso estudo, cita-se primeiramente a pequena amostra, o que dificultou análises sobre os fatores que determinam as escolhas de cada especialidade. Além disso, não foi possível realizar análises sobre associações entre as diversas variáveis, o que pode ter se dado pelo desenho do estudo, necessitando de estudos maiores para análises mais robustas.

\section{CONCLUSÃO}

Neste estudo, os fatores mais importantes para a escolha da segunda especialidade após a residência em clínica médica foram trabalho em ambiente ambulatorial, acompanhamento de pacientes por longo período e mais contato com pacientes. Mais estudos são necessários para tecer correlações entre os fatores de escolha com a especialidade escolhida, a fim de entender melhor os processos que levam à decisão sobre realizar uma segunda especialidade após a clínica médica.

\section{CONTRIBUIÇÃO DOS AUTORES}

Marco Felipe Macêdo Alves e Norma Arteiro Filgueira participaram da elaboração da pesquisa, da coleta e formatação dos dados, da revisão bibliográfica e da produção do manuscrito. Jardelina Brena Rocha Leite participou da coleta de dados e da elaboração e revisão do artigo.

\section{CONFLITO DE INTERESSES}

Declaramos não haver conflito de interesses.

\section{FINANCIAMENTO}

Declaramos não haver financiamento.

\section{REFERÊNCIAS}

1. Conselho Regional de Medicina de São Paulo. Parecer: Consulta no 60.960. Brasília: Cremesp; 2010 [access in 28 jun 2020]. Available from: http://www.cremesp.org.br/?siteAcao $=$ Pareceres, dif $=$ s, ficha $=1, i d=9701$, tipo $=$ PARECER, orgao $=$ Conselho $\% 2520$ Region al\%2520de\%2520Medicina\%2520do\%2520Estado\%2520de\%2520S\% $25 \mathrm{E} 30 \% 2520$ Paulo, numero $=60960$, situacao $=$,data $=23-11-2010$ Brazilian Portuguese.

2. Comissão Nacional de Residência Médica. Resolução CNRM nº 02/2006. Dispõe sobre requisitos mínimos dos Programas de Residência Médica e dá outras providências. Brasília: CNRM; 2006 [access in 28 jun 2020]. Available from: http://portal.mec.gov.br/dmdocuments/ resolucao02_2006.pdf. 
3. Martins JB, Rodriguez FP, Coelho ICMM, Silva EM. Fatores que influenciam a escolha da especialização médica pelos estudantes de Medicina em uma instituição de ensino de Curitiba (PR). Rev Bras Educ Med. 2019;43(2):1528. doi: 10.1590/1981-52712015v43n2rb20180158.

4. West $\mathrm{CP}$, Dupras DM. General medicine vs subspecialty career plans among internal medicine residents. JAMA. 2012;308(21):2241-7. doi: 10.1001/jama.2012.47535.

5. Yang J, Singhal S, Weng Y, Bentley JP, Chari N, Liu T, et al. Timing and predictors of subspecialty career choice among internal medicine residents: a retrospective cohort study. J Grad Med Educ. 2020 Apr 10;12(2):212-6. doi: 10.4300/JGME-D-19-00556.1.

6. Horn L, Tzanetos K, Thorpe K, Straus SE. Factors associated with the subspecialty choices of internal medicine residents in Canada. BMC Med Educ. 2008;8(1):1-8. doi: 10.1186/1472-6920-8-37.

7. Diehl AK, Kumar V, Gateley A, Appleby JL, O'Keefe ME. Predictors of final specialty choice by internal medicine residents. J Gen Intern Med. 2006;21(10):1045-9. doi: 10.1111\%2Fj.1525-1497.2006.00556.x.

8. Costa JRB, Romano VF, Costa RR, Gomes AP, Alves LA, Siqueira-Batista R. A transformação curricular e a escolha da especialidade médica. Rev Bras Educ Med. 2014;38(1):47-58. doi:10.1590/S0100-55022014000100007.

9. Ministério da Educação. Matrizes de Competências em Clínica Médica. Brasília; MEC; 2018 [access in 11 feb 2021]. Available from: http://portal. mec.gov.br/index.php?option=com_docman\&view=download\&ali as $=119741-13$-matriz-de-competencias-em-clinica-medica\&category slug=agosto-2019-pdf\&ltemid=30192.

10. Armstrong K, Keating NL, Landry M, Crotty BH, Phillips RS, Selker HP. Academic general internal medicine: a mission for the future. J Gen Intern Med. 2013;28(6):845-51. doi: 10.1007/s11606-013-2334-3.

11. Scheffer $M$, Cassenote $A$, Guerra A, Guilloux AGA, Brandão APD, Miotto BA, et al. Demografia médica no Brasil 2020. São Paulo: FMUSP, CFM; 2020 [access in 08 feb 2021]. Available from: https://www.fm.usp.br/fmusp/ conteudo/DemografiaMedica2020_9DEZ.pdf.
12. Garibaldi RA, Popkave C, Bylsma W. Career plans for trainees in internal medicine residency programs. Acad Med. 2005;80(5):507-12. doi: 10.1097/00001888-200505000-00021.

13. Scheffer MC, Dal Poz MR. The privatization of medical education in Brazil: trends and challenges. Hum Resour Health. 2015;13(1):1-10. doi: 10.1186/ s12960-015-0095-2.

14. Almeida Júnior VP, Pedrosa PAE. Fundo de Financiamento Estudantil (Fies): vicissitudes e desafios. Radar. 2018;58(1)37-41 [access in 11 feb 2021]. Available from: http://repositorio.ipea.gov.br/bitstream/11058/8839/1/ Radar_n58_fundo.pdf.

15. Cabral Filho WR, Ribeiro VMB. A escolha precoce da especialidade pelo estudante de Medicina: um desafio para a educação médica. Rev Bras Educ Med. 2004;28(2):133-44. doi: 10.1590/1981-5271v28.2-018.

16. Lucchetti ALG, Lucchetti G. Medical job market in the state of São Paulo: investigation of job proposals in the site "Banco de Empregos Médicos". Rev Soc Bras Clín Méd. 2014;12(2):137-44 [access in 11 feb 2021]. Available from: http://files.bvs.br/upload/S/1679-1010/2014/v12n2/a4181.

17. Revista da Sociedade Brasileira de Clínica Médica e a valorização do clínico [editorial]. Rev Soc Bras Clín Méd. 2018;16(2):75-6 [access in 11 feb 2021] Available from: http://www.sbcm.org.br/ojs3/index.php/rsbcm/article/ view/334/302.

18. Gouveia PAC, Ribeiro Neta MHC, Aschoff CAM, Gomes DP, Silva NAF, Cavalcanti HAF. Factors associated with burnout syndrome in medical residents of a university hospital. Rev Assoc Med Bras. 2017;63(6):504-11. doi: 10.1590/1806-9282.63.06.504.

19. Shanafelt TD, Bradley KA, Wipf JE, Back AL. Burnout and self-reported patient care in an internal medicine residency program. Ann Intern Med. 2002;136(5):358-67. doi: 10.7326/0003-4819-136-5-200203050-00008.

20. Rodrigues H, Cobucci R, Oliveira A, Cabral JV, Medeiros L, Gurgel K, et al. Burnout syndrome among medical residents: a systematic review and meta-analysis. PLoS ONE. 2018;13(11):e0206840. doi: 10.1371/journal. pone.0206840. 